\title{
NOUVELLE
}

\section{Effets locaux du BDNF et plasticité synaptique: vers une spécificité synaptique}

Serge Marty
> Les neurotrophines qui regroupent le nerve growth factor (NGF), le brain-derived neurotrophic factor (BDNF), la neurotrophine 3 (NT-3) et la neurotrophine 4/5 (NT-4/5), exprimées dans de nombreuses régions du système nerveux central (SNC) et en périphérie, assurent la survie des neurones du système nerveux, en particulier périphérique, au cours du développement [1] $(\rightarrow)$. Mais, outre leurs effets sur la survie cellulaire, elles pourraient également intervenir dans la formation des circuits neuronaux. La synthèse et la libération du NGF et du BDNF par les neurones centraux sont sous le contrôle de l'activité neuronale, et les neurotrophines peuvent en retour moduler les transmissions GABAergiques et glutamatergiques $[2,3]$. D'où I'hypothèse d'un rôle important des neurotrophines, et en particulier du BDNF qui est très largement exprimé dans le SNC, dans la plasticité synaptique, c'est-à-dire dans les modifications morphologiques et physiologiques des synapses en réponse à des changements d'activité neuronale [2]. Des expérimentations utilisant l'application de BDNF exogène, l'injection de récepteurs tronqués du BDNF, ou des animaux dont le gène codant pour le BDNF a été inactivé par recombinaison homologue, ont confirmé cette hypothèse. Ainsi, le BDNF a été impliqué dans la potentialisation à long terme des synapses excitatrices de l'hippocampe [4]. Dans un modèle de plasticité synaptique au cours du développement, celui des colonnes de dominance ocu-
Inserm U.106,

Bâtiment Pédiatrie, Hôpital Pitié-Salpêtrière, 47, boulevard de l'Hôpital, 75651 Paris Cedex 13, France. laire*, le BDNF a été impliqué dans la séparamarty@chups.jussieu.fr
ADNc codant pour le BDNF et un ADNc codant pour la red fluorescent protéine (pour visualiser les neurones surexprimant le BDNF). Lorsqu'un neurone exprimant seulement la GFP est situé à proximité d'un neurone exprimant le BDNF, son arborisation dendritique est analysée 36 heures après transfection. La tion en colonnes des axones thalamiques dans le cortex visuel [5]. Mais le site de libération du BDNF, pré- ou post-synaptique, doit encore être élucidé $[6,7]$, et le rayon d'action du BDNF une fois libéré reste peu connu. En effet, une des questions actuellement débattues est de savoir si le BDNF peut intervenir dans un court rayon d'action: s'il est libéré localement, il pourrait agir spécifiquement sur les synapses actives, impliquant cette neurotrophine dans les processus de stabilisation ou de potentialisation sélective de synapses. Deux études récentes abordent ce problème.

Horch et Katz [8] ont étudié l'effet de la surexpression de BDNF par un neurone sur la morphologie des arbres dendritiques des neurones adjacents (Figure lA). L'expérience a été faite dans des tranches de cortex visuel de furet en culture. Les neurones sont transfectés à l'aide d'un canon à gènes et, dans une même tranche, ils peuvent être transfectés soit par l'ADNc codant pour la seule GFP (green fluorescent protein), qui permet une bonne visualisation de leur morphologie, soit co-transfectés par un

\footnotetext{
* Les colonnes de dominance oculaire caractérisent le cortex visuel, organisé en une véritable carte rétinotopique composée de modules juxtaposés, ou intriqués, de colonnes indépendantes. Ces colonnes sont des bandes de tissu cortical alternativement occupées par des afférences issues de l'œil gauche ou de l'œil droit. Elles sont particulièrement marquées au niveau de la couche IV du cortex, qui reçoit les terminaisons des afférences du corps genouillé latéral, et où les neurones sont exclusivement activés par la stimulation de l'un ou l'autre œil.
}

reconstruction des arbres dendritiques a permis de déceler une augmentation du nombre de points de branchement et de longueur des dendrites orientés vers le neurone surexprimant le BDNF, mais uniquement de ces dendrites. De plus, cette modification est très locale et ne concerne que les parties distales du dendrite ayant croisé les dendrites du neurone surexprimant le BDNF, jusqu'à une distance de 4,5 $\mu \mathrm{m}$. Cet effet n'est pas observé si on fait exprimer par les neurones cibles $\mathrm{GFP}^{+}$un récepteur tronqué $d u$ BDNF qui agit comme un dominant négatif, indiquant la nécessité d'une action directe du BDNF sur les dendrites. Ces résultats montrent que le BDNF peut agir de façon locale sur les arborisations dendritiques, en favorisant leur croissance et leur complexité.

La deuxième étude [9] montre qu'une source de BDNF n'affecte les propriétés électrophysiologiques des terminaisons pré-synaptiques que si elle est située à proximité de ces terminaisons (Figure 1B). Dans des co-cultures de neurones spinaux et de myocytes de xénope, l'application pendant 10 minutes d'une bille couverte de BDNF au contact d'un axone entraîne une augmentation de la fréquence des courants miniatures postsynaptiques excitateurs, mais uniquement si la synapse entre cet axone et un myocyte est située à une distance maximale de $60 \mu \mathrm{m}$ de la bille. Cette distance maximale ne change pas même si on augmente la quantité locale du facteur neurotrophique en plaçant plusieurs billes au 
contact de l'axone, indiquant que l'effet obtenu ne résulte pas de l'établissement d'un gradient de BDNF. II s'agirait plutôt de l'activation intra-axoplasmique de cascades moléculaires consécutive à une augmentation du calcium dans une portion de l'axone longue de $40 \mu \mathrm{m}$ à partir de la bille. L'effet du BDNF persiste même après une section de l'axone le séparant du corps cellulaire: mais, de façon surprenante, cet effet est bloqué lorsqu'un inhibiteur de la synthèse protéique est injecté dans le neurone deux heures avant l'application de BDNF, indiquant la nécessité d'une synthèse protéique axonale. Celle-ci n'est cependant pas nécessaire au moment de l'application du BDNF, 45 minutes de pré-incubation avec des inhibiteurs de la synthèse protéique ne suffisant pas à bloquer les effets du BDNF.

Ces deux études montrent que le BDNF peut effectivement avoir des effets locaux sur des portions restreintes d'axones ou de dendrites. La restriction spatiale des effets du BDNF n'est cependant pas suffisante pour permettre une spécificité synaptique. En effet, dans le SNC, le rayon d'action du BDNF défini dans ces travaux couvre de nombreuses synapses, probablement en raison $\mathrm{du}$ mode d'application du BDNF dans ces modèles expérimentaux, qui entraîne une exposition continue à la neurotrophine. In vivo, la libération de BDNF sous l'effet d'une augmentation de l'activité neuronale pourrait agir de façon très ponctuelle et donc sur un domaine de taille encore plus restreinte. II reste donc encore à élucider dans quelle mesure l'effet de stimulations physiologiques, qui provoquent une libération du BDNF endogène restreinte dans le temps et dans l'espace, pourrait agir de façon spécifique sur les synapses actives. Par ailleurs, l'histoire ne pourra pas être complète si l'on ne détermine pas avec précision la part de l'action pré-synaptique [9] et post-synaptique [8] dans les effets du BDNF. $\diamond$

Local response to neurotrophic factors

\section{RÉFÉRENCES}

1. Lewin GR, Barde YA. Physiology of the neurotrophins. Annu Rev Neurosci 1996; 19: 289-317.

2. Thoenen H. Neurotrophins and neuronal plasticity. Science 1995; 270 : 593-8.

3. Berninger $B$, Poo MM. Fast actions of neurotrophic factors. Curr Opin Neurobiol 1996; 6: 324-30.

4. Minichiello L, Calella AM, Medina DL, Bonhoeffer T, Klein R, Korte $M$. Mechanism of TrkB- mediated hippocampal long-term potentiation. Neuron 2002; 36: 121-37.

5. Berardi N, Maffei L. From visual experience to visual function: roles of neurotrophins. J Neurobiol 1999; 41: 119-26.

6. Hartmann M, Heumann R, Lessmann V. Synaptic secretion of BDNF after high-frequency stimulation of glutamatergic synapses. EMBO J 2001; 20 : 5887-97.

7. Balkowiec A, Katz DM. Cellular mechanisms regulating activitydependent release of native brain-derived neurotrophic factor from hippocampal neurons. J Neurosci 2002; 23 : 10399-407.

8. Horch HW, Katz LC. BDNF release from single cells elicits local dendritic growth in nearby neurons. Nat Neurosci 2002; 5 : 1177-84.

9. Zhang XH, Poo MM. Localized synaptic potentiation by BDNF requires local protein synthesis in the developing axon. Neuron 2002; 36 : 675-88.

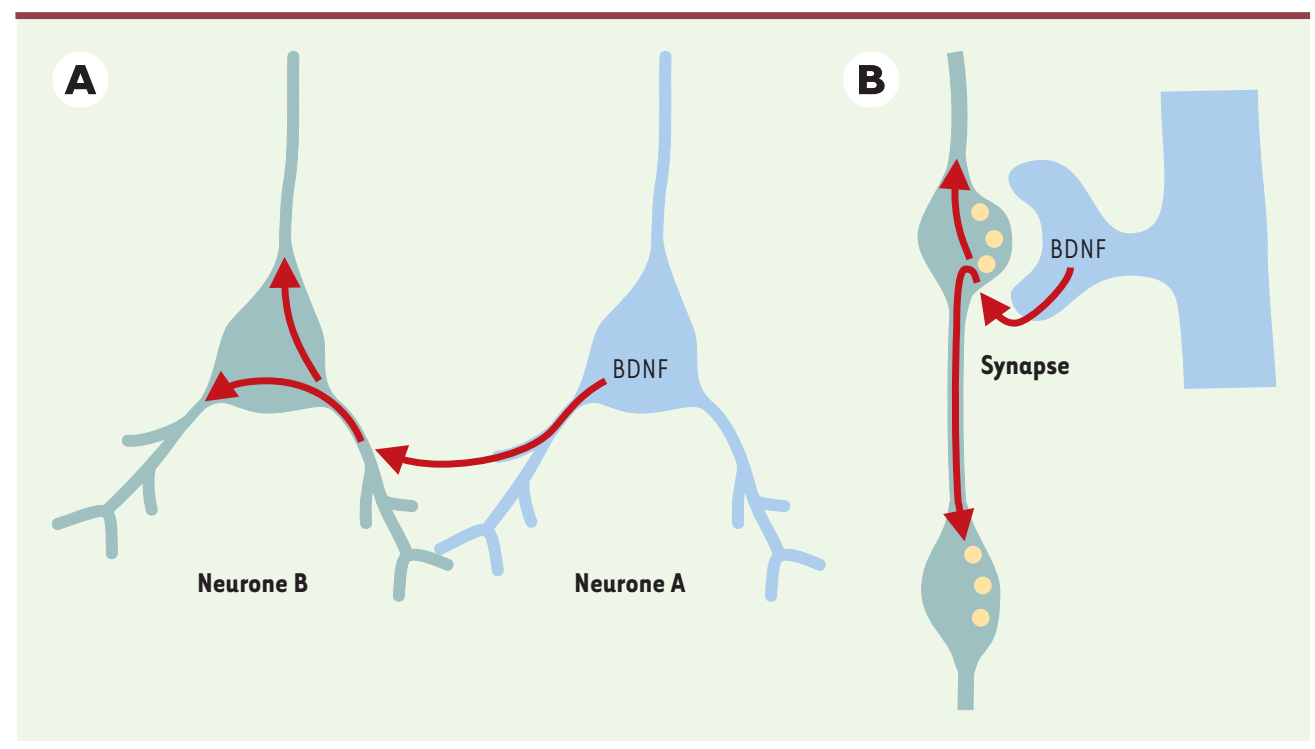

Figure 1. Effets du BDNF sur les dendrites (A) ou les axones (B). A. Le BDNF libéré par un neurone pourrait agir localement sur une région de l'arborisation dendritique du neurone adjacent, ou modifier l'ensemble des branches dendritiques. B. La libération du BDNF par l'élément post-synaptique pourrait agir spécifiquement sur la terminaison pré-synaptique adjacente, ou entraîner également des modifications de l'axone à distance. 